\title{
Effect of Bubble Coalescence on Material Removal Rate in Electrical Discharge Machining Process
}

\author{
Shinya Hayakawa*, Soichiro Yamada*, Fumihiro Itoigawa*, Takashi Nakamura*, \\ Tomohiko Kitamura**, Masami Yamanaka** \\ (Received September 10, 2010)
}

* Department of Mechanical Engineering, Nagoya Institute of Technology, Nagoya 466-8555, Japan

** Lubricants Research Laboratory, Idemitsu Kosan Co. Ltd., Ichihara 299-0107, Japan

\begin{abstract}
This paper investigates the ease of bubble coalescence of the machining liquid and the medium at the discharge ignition location in electrical discharge machining (EDM) process in order to discuss the effect of the properties of the machining liquid on the material removal rate. Bubble coalescence, which is one of the properties of a machining liquid, was observed using a video camera, and the medium at the discharge ignition location, i.e., whether a discharge occurs in EDM oil or in bubbles, was determined using a characteristic of the waveform of the discharge current. It is found for a continuous EDM process that a high proportion of discharges occur near the boundaries of previously existing bubbles, whereas few discharges occur in the interior of bubbles. It is also found that reducing the bubble coalescence will improve the material removal rate.
\end{abstract}

Key words: electrical discharge machining, bubble, coalescence, material removal rate

\section{INTRODUCTION}

In electrical discharge machining (EDM), the properties of the machining liquid affect the material removal rate. Kitamura et al. ${ }^{1)}$ reported that the heat transfer coefficient of the machining liquid affects the removal efficiency of molten material at the discharge location because it reflects the intensity of nucleate boiling of the machining liquid. Miki $^{2)}$ reported that when the electrical resistivity of the machining liquid is lower, the material removal rate increases. The machining liquid also affects the material removal rate through the generation of bubbles. Takeuchi and Kunieda ${ }^{3)}$ calculated the flow field of a machining liquid in the gap space and reported that most of the gap space is filled with bubbles. Yoshida and Kunieda ${ }^{4)}$ reported that material removal from the discharge location occurs even when a discharge is generated in air, but almost all the debris particles are reattached on the electrode surface. The authors ${ }^{5)}$ observed the flying debris particles scattered from the discharge point using a high-speed video camera and reported that scattered debris particles follow the expanding bubble boundary, and after entering the machining liquid, debris particles float in the liquid near the bubble boundary. The penetration depth from the bubble boundary into liquid was about $1 \mathrm{~mm}^{5)}$. We also reported that the scattering of debris particles can be observed even when a single pulse discharge is generated in a bubble ${ }^{6}$. However, for the actual EDM process, the diameter of bubbles existing in the gap space and the proportion of pulse discharges generated in bubbles are not known. In this paper, the coalescence of bubbles in the gap space and the medium at the discharge ignition location in EDM process are investigated in order to discuss the effect of the properties of the machining liquid on the material removal rate.

\section{COALESCENCE OF BUBBLES}

\subsection{Experimental Method}

In order to investigate the ability of bubbles to coalesce and compare the ability between different types of EDM oil, the behavior of the bubble coalescence is experimentally observed. The experimental method is shown in Fig. 1. A transparent resin plate is set in a machining tank and EDM oil is poured into the tank. Bubbles of air are generated below the transparent plate using a syringe, and the bubbles rise to the underside of the transparent plate and remain there. The behavior of bubble coalescence is observed using a video camera. Although this experiment is not carried out in a parallel flat gap space and bubbles are composed of air (the components of bubbles are different from those generated in the actual EDM process), the ability of bubble to coalesce could be compared among different types of machining liquid by this method, because the ability of bubbles to coalesce is largely affected by the physical properties of the liquid such as surface tension.

Two types of commercially available EDM oil were used for this experiment. The properties of the oils are listed in Table 1. Under the machining 


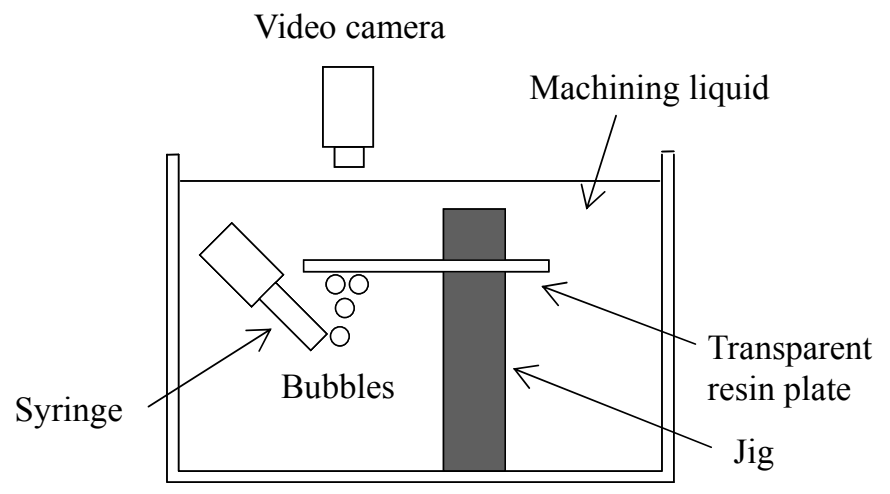

Fig. 1 Experimental method

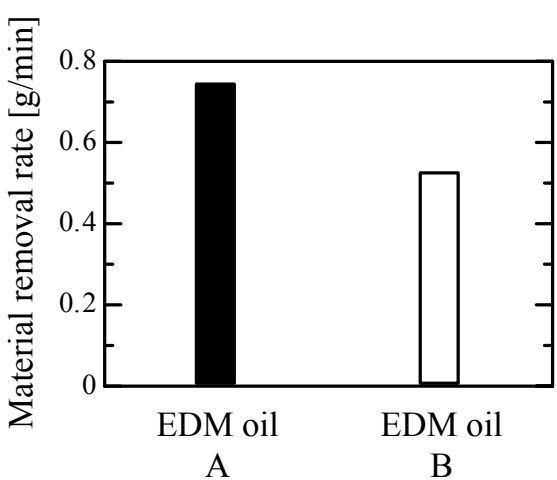

Fig. 2 Material removal rate
Table 1 Properties of EDM oils

\begin{tabular}{c|c|c}
\hline & EDM oil A & EDM oil B \\
\hline Base oil & $\begin{array}{c}\text { Synthetic } \\
\text { oil }\end{array}$ & $\begin{array}{c}\text { Synthetic } \\
\text { oil }\end{array}$ \\
\hline $\begin{array}{c}\text { Kinematic viscosity } \\
\left(40^{\circ} \mathrm{C}\right)\left[\mathrm{mm}^{2} / \mathrm{s}\right]\end{array}$ & 2.2 & 2.6 \\
\hline $\begin{array}{c}\text { Density }\left[\mathrm{g} / \mathrm{cm}^{3}\right] \\
\text { Volumetric resistivity } \\
{[\Omega \mathrm{m}]}\end{array}$ & 0.78 & 0.79 \\
\hline \begin{tabular}{c} 
Ve12 \\
\hline
\end{tabular}
\end{tabular}

conditions listed in Table 2, the material removal rate of type A oil is greater than that of type B oil ${ }^{1)}$, as shown in Fig. 2. Since the discharge frequency, gap width, material removal volume by a single pulse discharge, bubble expansion and contraction observed using a high-speed video camera, and proportion of the components of bubbles analyzed using a gas chromatography are almost the same among the two cases, the difference of the material removal rate is considered to be affected by reattaching of scattered debris particles on the electrode surface.

\subsection{Observed Results}

Figure 3 shows the observed time variation of the bubble coalescence. In the case of type A oil, bubbles $1 \mathrm{~mm}$ or more in diameter got together on the undersurface of the plate but rarely coalesced, as shown in Fig. 3(a). On the other hand, in the case of type B oil, bubbles $1 \mathrm{~mm}$ or more in diameter coalesced one after another and the diameter of the coalesced bubbles became large, as shown in Fig. 3(b). Since the ability of bubbles to coalesce is affected by the physical properties of the liquid such
Table 2 Machining conditions

\begin{tabular}{c|c}
\hline Tool electrode & Copper $(\phi 20 \mathrm{~mm})$ \\
\hline Workpiece & Mild steel $(\phi 20 \mathrm{~mm})$ \\
\hline Applied voltage & $90 \mathrm{~V}$ \\
\hline Polarity & Tool electrode $(+)$ \\
\hline Discharge current & $35 \mathrm{~A}$ \\
\hline Discharge duration & $256 \mu \mathrm{s}$ \\
\hline Pulse interval & $256 \mu \mathrm{s}$ \\
\hline
\end{tabular}

as surface tension, the difference of the behavior of bubble coalescence should be similar in the actual EDM process.

\section{MEDIUM AT DISCHARGE IGNITION LOCATION}

\subsection{Method of Determining Medium}

In order to investigate the effect of bubble coalescence on the material removal rate, a method of determining the medium at the discharge ignition location, i.e., whether a discharge occurs in the dielectric liquid or in a bubble, is necessary. Therefore, a method using a characteristic of the waveform of the discharge current ${ }^{7)}$ was developed in this paper.

Figure 4 shows examples of waveforms of the discharge current when a pulse discharge was generated in a gas (air) and in a dielectric liquid (EDM oil). For the case of a gas, the rising edge of the waveform of the discharge current is rectangular, whereas it is rounded for the case of a dielectric liquid. It is considered that this characteristic of 


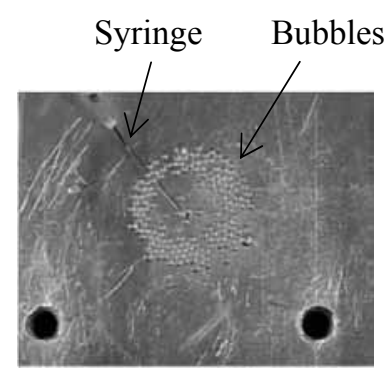

$5 s$

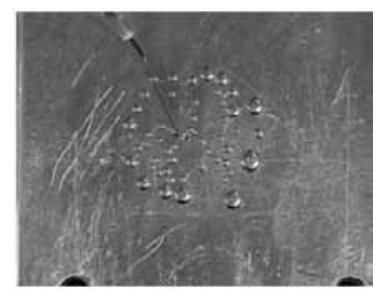

$5 \mathrm{~s}$

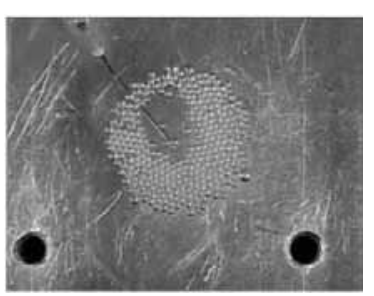

$10 \mathrm{~s}$

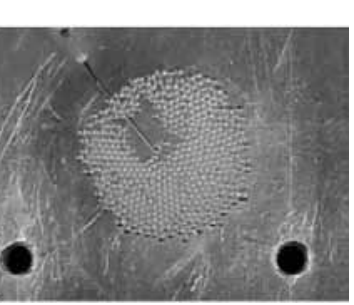

$15 \mathrm{~s}$

(a) EDM oil A

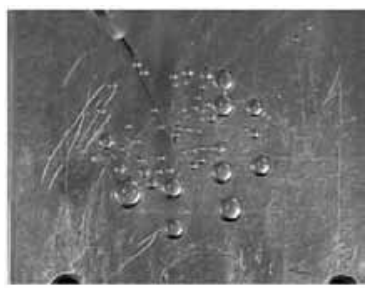

$10 \mathrm{~s}$

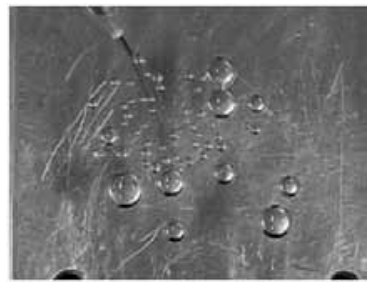

$15 \mathrm{~s}$

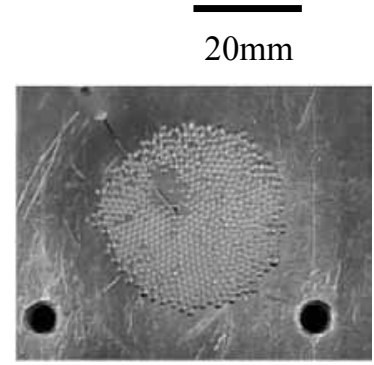

$20 \mathrm{~s}$

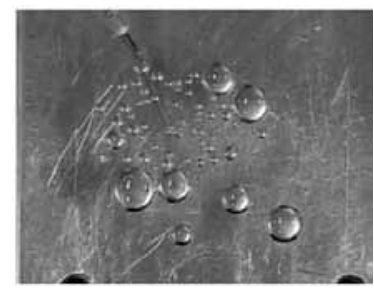

$20 \mathrm{~s}$

(b) EDM oil B

Fig. 3 Time variation of bubble coalescence (top view)

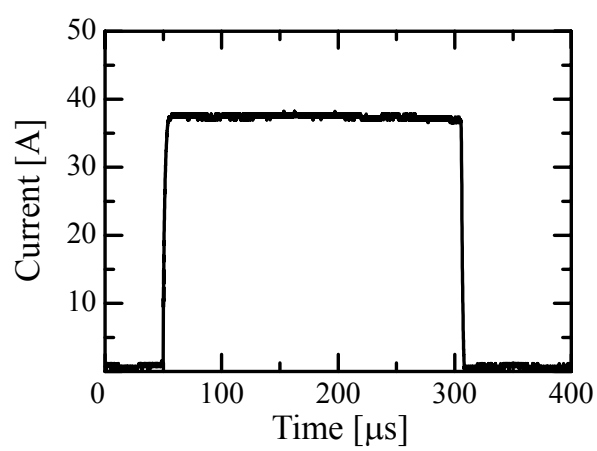

(a) Discharge in gas

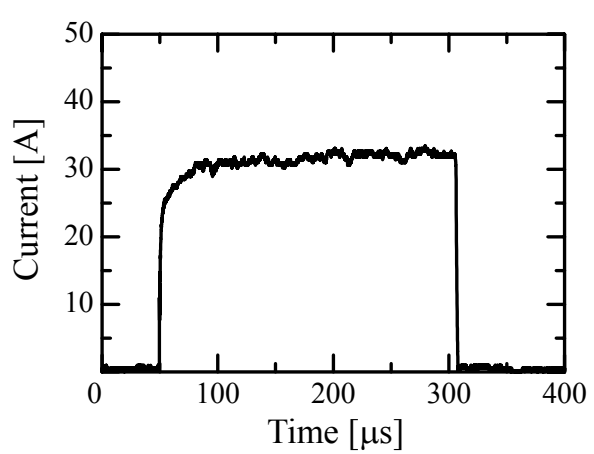

(b) Discharge in liquid

Fig. 4 Waveforms of discharge current

the waveform reflects the difference in the plasma formation and expansion rate, namely, plasma formation and expansion in a gas is rapid, whereas in a liquid it is relatively slow. On the basis of this characteristic of the waveform, the discharge current rise time can therefore be used to determine the medium at the discharge ignition location for each pulse discharge. In this paper, the discharge current rise time is defined as the time at which the discharge current reaches $95 \%$ of the peak value.

\subsection{Results for Continuous EDM Process}

The discharge current rise time was investigated during the continuous EDM process for the two types of EDM oil listed in Table 1. In this experiment, continuous EDM process is carried out and the waveforms of 400 pulse discharges are randomly sampled. The experimental conditions are the same as those listed in Table 2.

The obtained relative frequency distribution of the discharge current rise time for each EDM oil are shown in Fig. 5. The results for the case of a single pulse discharge generated in each EDM oil and that measured in air are also shown in Fig. 5. The experimental results show that the discharge current rise time measured for the continuous EDM process in EDM oil is distributed from $10 \mu \mathrm{s}$ to $60 \mu \mathrm{s}$, whereas that for a single pulse discharge in EDM oil is distributed from $20 \mu \mathrm{s}$ to $100 \mu \mathrm{s}$. In contrast, the discharge current rise time measured in air is less than $5 \mu$ s for all pulses. The results are similar for type A oil and type B oil. It is found that the medium at the discharge ignition location in the 


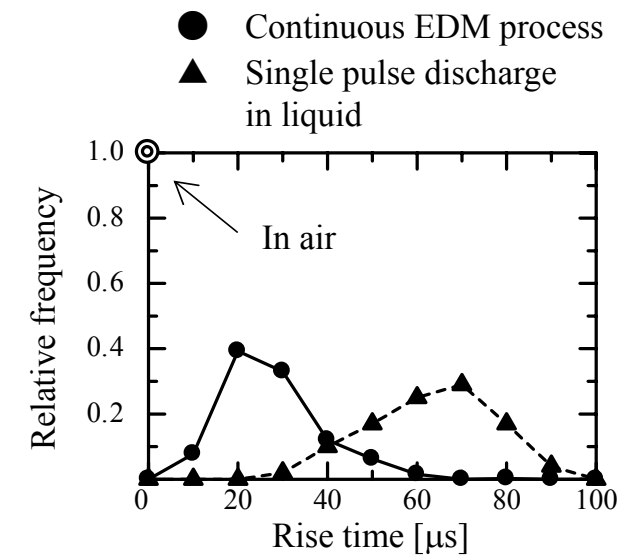

(a) EDM oil A

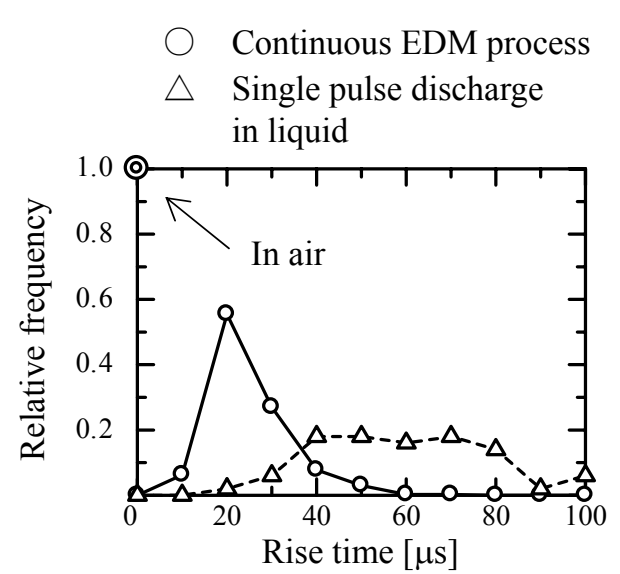

(b) EDM oil B

Fig. 5 Relative frequency distribution of discharge current rise time

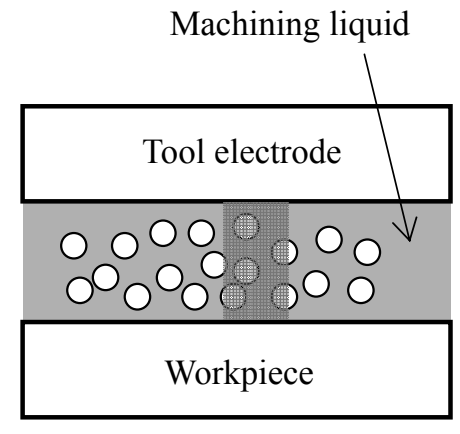

(a) Model 1

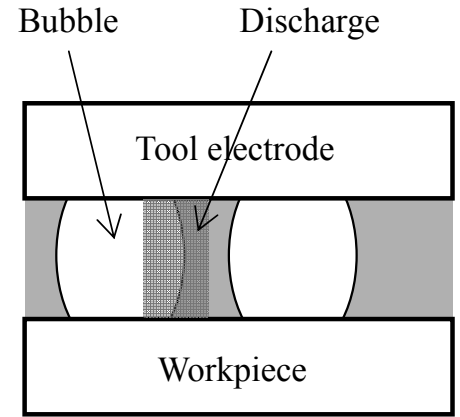

(b) Model 2

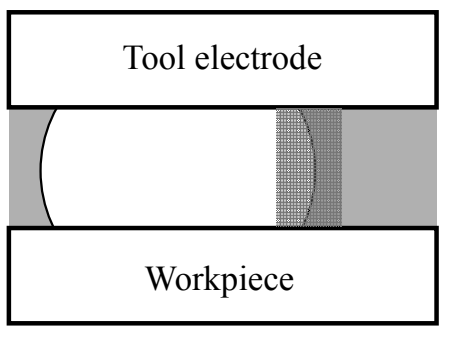

(c) Model 3

Fig. 6 Models of coexistence of liquid and bubble (side view)

continuous EDM process cannot be classified into a liquid or gas. This result indicates that the medium at the discharge ignition location is coexistence of EDM oil and bubbles.

\subsection{Models of Coexistence of Liquid and Bubble}

Yoshida and Kunieda ${ }^{4)}$ observed the distribution of scattered debris particles for a single pulse discharge in a liquid and reported that debris particles are mainly distributed at the boundary of bubble formed by the discharge. The authors ${ }^{5)}$ also reported that scattered debris particles follow the expanding bubble boundary, and after entering the machining liquid, debris particles float in the liquid near the bubble boundary. Mori and Kunieda ${ }^{8)}$ observed the behavior of debris particles existing in the gap space and reported that when an electric field is applied between the anode and cathode, debris particles move back and forth between them. Debris particles also form bridges between the anode and cathode, which lead to electrical breakdown. Kunieda and Nakashima ${ }^{9)}$ reported that in a series of pulse discharges, numerous chains of debris particles grow over the working surface, and the debris particle concentration affects the discharge location more dominantly than the gap width.

Summarizing these reported findings, it is thought that electrical breakdown tends to occur at bubble boundaries in continuous EDM process. Therefore, the following three models can be considered as the probable state of the coexistence of EDM oil and bubbles, as shown in Fig. 6.

Model 1: A large number of bubbles with diameter much smaller than the gap width are suspended, as shown in Fig. 6(a), and a discharge occurs among them.

Model 2: Some bubbles exist whose volume is similar to that of a bubble generated by a pulse discharge and, therefore, bubbles with a diameter larger than the gap width are located close to each other, and a discharge occurs near the boundary of one of the bubbles, as shown in Fig. 6(b). In this model, it is considered that each bubble is generated 


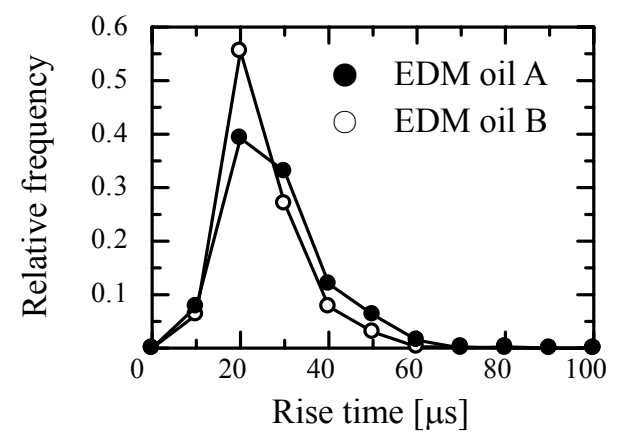

Fig. 7 Relative frequency distribution of discharge current rise time

by a pulse discharge and that bubbles do not coalesce with each other. Debris particles are assumed to be located near the boundaries of the bubbles.

Model 3: A small number of bubbles exist whose volume is a few times larger than that of a bubble generated by a pulse discharge and, therefore, bubbles with a diameter much larger than the gap width are located distantly from each other, and a discharge occurs near the boundary of a bubble, as shown in Fig. 6(c). Each bubble is considered to be formed by the coalescence of bubbles, each of which is generated by a pulse discharge. When the coalescence of bubbles occurs, debris particles previously located near the boundaries of the generated bubbles are assumed to be displaced to the boundary of the newly coalesced bubble.

The authors ${ }^{5)}$ observed the bubble expansion and contraction caused by a discharge generated in the EDM oil using a high-speed video camera. In the experiment, the discharge was generated in a parallel flat gap space of $50 \mu \mathrm{m}$ in gap width. The observed results show that the dimension of the bubble immediately after the electrical breakdown is larger than the gap width. Therefore, model 1 shown in Fig. 6(a) can be eliminated, and model 2 and model 3 are considered to be applicable to the continuous EDM process.

\subsection{Discussion}

In section 3.1, we attempted to classify pulse discharges into two categories: discharge in a dielectric liquid and discharge in a bubble. However, the experimental results shown in Fig. 5 indicate that the classification must be changed so as to consider a coexistence of liquid and bubble. Therefore, a discharge previously considered to be in a liquid is redefined as a discharge that is strictly generated in a liquid (i.e., far from bubbles), and a discharge previously considered to be in a bubble is redefined as a discharge that is generated in the interior of a bubble (i.e., far from the bubble boundary). Moreover, a discharge generated near bubble boundary is classified as one occurring in the coexistence of liquid and bubble.

The experimental results shown in Fig. 5 are once again discussed. Since the distribution of the discharge current rise time measured for continuous EDM process in EDM oil does not overlap with that measured in air, few pulse discharges should occur in the interior of bubbles (far from the bubble boundary). On the other hand, since the tail of the distribution for continuous EDM process overlaps with the result for single pulse discharge in a liquid, a certain proportion of pulse discharges should occur in the liquid (far from bubbles). However, the result that the distribution for continuous EDM process hardly overlaps with that for single pulse discharge in the liquid can be interpreted to mean that most of the pulse discharges occur near the bubble boundary.

Figure 7 shows a comparison of the discharge current rise times of type A oil and type B oil obtained in the continuous EDM process, each of which have been shown in Fig. 5. It can be seen that the distribution of type $\mathrm{B}$ oil is slightly more weighted in the direction of a small discharge current rise time. This result indicates that when type B oil is used, plasma can more rapidly formed and expand than when type A oil is used. Therefore, the state of the coexistence of liquid and bubble, namely, the bubble boundary, of type B oil is thought to be closer to that in the interior of the bubble. This will be discussed again in the next section.

\section{EFFECT OF BUBBLE COALESCENCE ON MATERIAL REMOVAL RATE}

In this section, the effect of bubble coalescence on the material removal rate is discussed, based on the experimental results shown in Fig. 3 and Fig. 7.

First, the correspondence between the oil type 


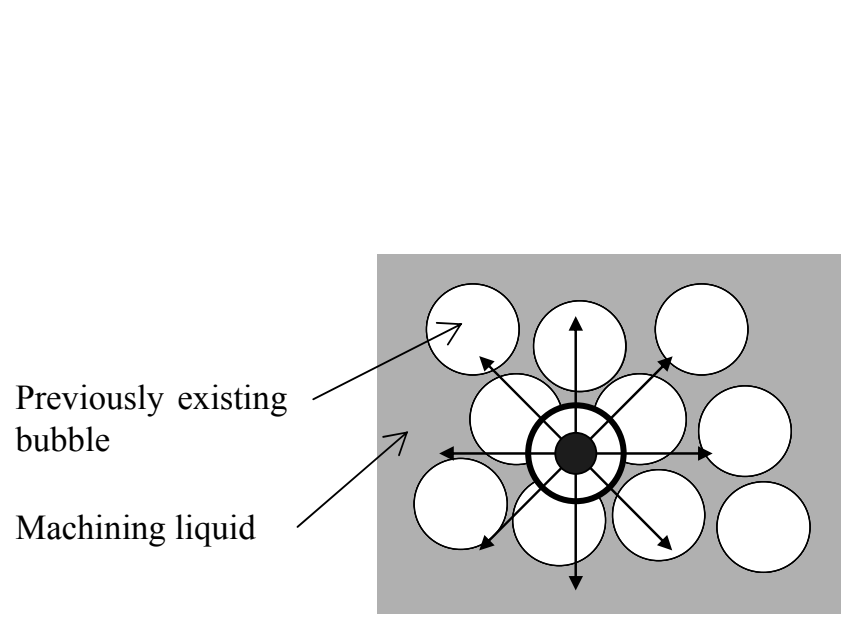

(a) EDM oil A
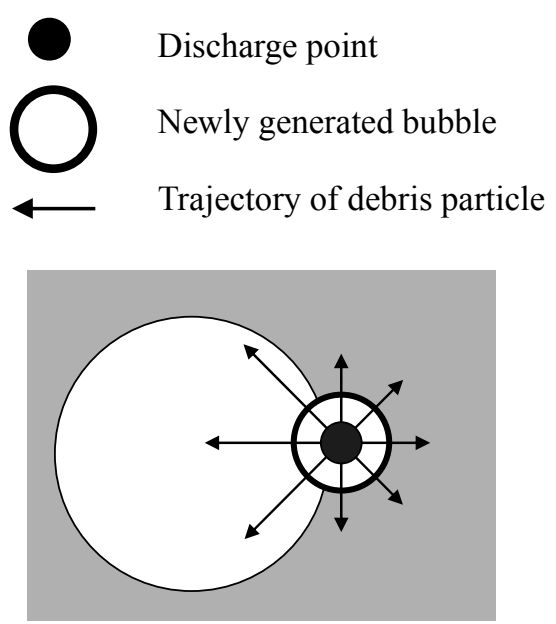

(b) EDM oil B

Fig. 8 Schematics of expected material removal phenomena (top view)

and the model of coexistence of liquid and bubble shown in Fig. 6 is determined. Since the bubbles in type A oil hardly coalesced, model 2 can be applied for type A oil. On the other hand, since the bubbles in type B oil tended to coalesce, model 3 can be applied for type B oil.

Next, the material removal phenomena for the case of type A oil are discussed using model 2 . Figure 8(a) shows a schematic of the expected phenomena. If previously existing bubbles have gathered together but have not coalesced, then there is machining liquid (liquid film) between the bubbles. When a discharge occurs at a bubble boundary, newly generated debris particles are projected through the newly generated and previously existing bubbles. In this case, the scattered debris particles also pass through the machining liquid existing between the bubbles. Since the density and heat capacity of liquid are much greater than those of gas, and latent heat of evaporation of liquid also contributes to cooling the debris particles, cooling of the debris particles by liquid is considered to be much greater than that by gas. Therefore, the debris particles will not reattach on the surface of the electrode. Then, the efficiency of material removal is expected to be high.

Next, the material removal phenomena for the case of type B oil are discussed using model 3 . Figure 8(b) shows a schematic of the expected phenomena. There is a large coalesced bubble existing in the gap space. When a discharge occurs near the bubble boundary, newly generated debris particles are scattered in all directions. If a debris particle is projected away from the previously existing large bubble, it will pass through the newly generated bubble and enter the machining liquid. In this case, the particle is expected to be removed without reattaching on the surface of the electrodes. On the other hand, if a debris particle is projected toward the interior of the previously existing large bubble, it will remain within the bubble without passing through the machining liquid. In this case, the particle will reattach on the surface of the electrodes and will not be removed. Therefore, the efficiency of material removal for the case of type B oil is anticipated to be low compared with that for type A oil, even if a similar volume of material is scattered from the discharge point.

The above consideration agrees qualitatively with the measured material removal rate shown in Fig. 2. Therefore, it can be concluded that bubble coalescence is one of the factors affecting the material removal rate, and reducing the bubble coalescence will improve the material removal rate.

\section{CONCLUSIONS}

In this paper, the effect of bubble coalescence on the material removal rate in EDM process was investigated. The following conclusions were obtained.

(1) Whether a discharge occurs in a dielectric liquid or in a gas can be determined from the discharge current rise time. Under the conditions used in this paper, when a discharge occurs in a gas, the discharge current rise time is less than $5 \mu \mathrm{s}$, whereas that for a discharge generated in a EDM oil is distributed from $20 \mu \mathrm{s}$ to $100 \mu \mathrm{s}$.

(2) In the actual EDM process, a high proportion of 
discharges occur near the boundaries of previously existing bubbles, whereas few discharges occur in the interior of bubbles.

(3) Bubble coalescence is one of the factors affecting the material removal rate, and reducing the bubble coalescence will improve the material removal rate.

\section{REFERENCES}

1) Tomohiko Kitamura, Ryoichi Okuda, Shinya Hayakawa, Fumihiro Itoigawa, Takashi Nakamura: Effect of Machining Oil Properties on EDM Performance, Proceedings of World Tribology Congress 2009, (2009) p.500.

2) Shinsuke Miki: Influence of Machining Liquid on Machining Properties of EDM, Proceedings of 2004 Annual Meeting of Japan Society of Electrical Machining Engineers, (2004) pp.11-12.

3) Haruka Takeuchi, Masanori Kunieda: Effects of Volume Fraction of Bubbles in Discharge Gap on Machining Phenomena of EDM, Proceedings of 15th International Symposium on Electro Machining, (2005) pp.63-68.

4) Masahiro Yoshida, Masanori Kunieda: Study on the Distribution of Scattered Debris Generated by a Single Pulse Discharge in EDM Process, International Journal of Electrical Machining,
No.3 (1998) pp.39-46.

5) Shinya Hayakawa, Teruya Doke, Fumihiro Itoigawa, Takashi Nakamura: Observation of Bubble Expansion and Flying Debris in Parallel Flat Gap Space in Electrical Discharge Machining, International Journal of Electrical Machining, No.14 (2009) pp.29-35.

6) Teruya Doke, Shinya Hayakawa, Fumihiro Itoigawa, Takashi Nakamura: Observation of Scattered Debris Generated by Pulse Discharge in Bubble in Electrical Discharge Machining, Proceedings of 5th International Conference on Leading Edge Manufacturing in 21st Century, (2009) pp.845-850.

7) Shinya Hayakawa, Yumika Sudo, Kenji Omiya, Atsushi Watanabe, Fumihiro Itoigawa, Takashi Nakamura: Machining Phenomena of Electrical Discharge Machining at Gas-Liquid Interface, International Journal of Electrical Machining, No.15 (2010) pp.1-9.

8) Masahiro Mori, Masanori Kunieda: Study on Behavior of Debris Particles in Gap Space in EDM Process, Proceedings of 1992 Spring Meeting of Japan Society for Precision Engineering, (1992) pp.279-280.

9) Masanori Kunieda, Takanobu Nakashima: Factors Determining Discharge Location in EDM, International Journal of Electrical Machining, No.3 (1998) pp.53-58. 